\title{
ARTICLE
}

Clinical Study

\section{Survival of patients with deficient mismatch repair metastatic colorectal cancer in the pre-immunotherapy era}

\author{
G. Emerens Wensink et al. (ID)
}

BACKGROUND: Metastatic colorectal cancer patients with deficient mismatch repair (dMMR mCRC) benefit from immunotherapy. Interpretation of the single-arm immunotherapy trials is complicated by insignificant survival data during systemic nonimmunotherapy. We present survival data on a large, comprehensive cohort of dMMR mCRC patients, treated with or without systemic non-immunotherapy.

METHODS: Two hundred and eighty-one dMMR mCRC patients ( $n=54$ from three prospective Phase 3 CAIRO trials; $n=227$ from the Netherlands Cancer Registry). Overall survival was analysed from diagnosis of mCRC (OS), from initiation of first-line (OS1) and second-line (OS2) systemic treatment. Cox regression analysis examined prognostic factors. As comparison for OS 2746 MMR proficient $\mathrm{mCRC}$ patients were identified.

RESULTS: Of 281 dMMR patients, $62 \%$ received first-line and $26 \%$ second-line treatment. Median OS was 16.0 months (13.8-19.6) with antitumour therapy and 2.5 months (1.8-3.5) in untreated patients. OS1 was 12.8 months (10.7-15.2) and OS2 6.2 months (5.4-8.9) in treated dMMR patients. Treated dMMR patients had a 7.6-month shorter median OS than pMMR patients.

CONCLUSION: Available data from immunotherapy trials lack a control arm with standard systemic treatment. Given the poor outcome compared to the immunotherapy results, our data strongly suggest a survival benefit of immunotherapy in dMMR mCRC patients.

British Journal of Cancer (2021) 124:399-406; https://doi.org/10.1038/s41416-020-01076-0

\section{BACKGROUND}

Approximately $5 \%$ of metastatic colorectal cancer (mCRC) patients have a tumour with deficient DNA mismatch repair (dMMR), also referred to as microsatellite instability. ${ }^{1}$ dMMR arises through germline mutations or epigenetic methylation and inactivation of the MMR pathway, resulting in insertions or deletions in tandem repetitive sequences in DNA, a hypermutated genome, and a strong immune infiltrate. ${ }^{2}$ Sporadic dMMR tumours frequently harbour a BRAFV600E mutation and sporadic dMMR patients have a worse prognosis compared to Lynch syndrome patients. ${ }^{2,3}$

A dMMR tumour status has prognostic and therapeutic implications for patients, with dMMR having a favourable impact on prognosis in early-stage CRC, but resulting in a worse prognosis in mCRC compared to proficient mismatch repair (pMMR) tumours. $^{1,4,5}$ Immune checkpoint inhibitor (ICI) trials have shown a durable response in pretreated dMMR mCRC patients. ${ }^{6-8}$ dMMR tumours are highly sensitive to $\mathrm{ICl}$ due to the high mutational load, immune infiltrate and immune checkpoint signalling. ${ }^{6}$ Overman demonstrated a 1 -year OS rate of $85 \%$ in dMMR mCRC patients, who were refractory to at least one systemic treatment line, upon treatment with nivolumab/ipilimumab. ${ }^{8} \mathrm{ICI}$ have been approved by the Food and Drug Administration (FDA) in dMMR mCRC patients beyond first-line treatment. However, the European Medicines Agency (EMA) has not approved ICI based on the lack of a standard control arm without immunotherapy in the $\mathrm{ICl}$ trials. For a better interpretation of published immunotherapy results, survival data beyond first-line in dMMR $\mathrm{mCRC}$ patients who did not receive immunotherapy are needed.

Due to the low incidence of dMMR among mCRC patients, data on survival in $\mathrm{dMMR} \mathrm{mCRC}$ patients receiving standard systemic treatments are scarce. ${ }^{1,3,9-12}$ Published data of survival beyond first-line treatment in a cohort of dMMR mCRC patients not receiving immunotherapy are highly needed. We report on the survival and factors affecting survival of a large cohort of dMMR mCRC patients.

\section{METHODS}

Study population

We analysed dMMR $\mathrm{mCRC}$ patients in two populations: population-based patients registered in the Netherlands Cancer Registry (NCR) managed by the Netherlands Comprehensive Cancer Organisation (IKNL), and trial-based patients from three prospective Phase 3 first-line clinical trials (CAIRO, ${ }^{13}$ CAIRO $2^{14}$ and CAIRO $\left.{ }^{15}\right)$. For trial-based patients, patient inclusion criteria, informed consent and study protocols for the trials were published previously. ${ }^{13-15}$ The privacy rights for patients were maintained.

Inclusion criteria for the current analysis were histologically proven $\mathrm{mCRC}$ with a dMMR tumour. Patients who received immunotherapy during the course of their disease were excluded. Clinical data of all newly diagnosed cancer patients in the

Correspondence: Miriam Koopman (M.Koopman-6@umcutrecht.nl)

Extended author information available on the last page of the article

Received: 12 March 2020 Revised: 24 August 2020 Accepted: 2 September 2020

Published online: 13 October 2020 


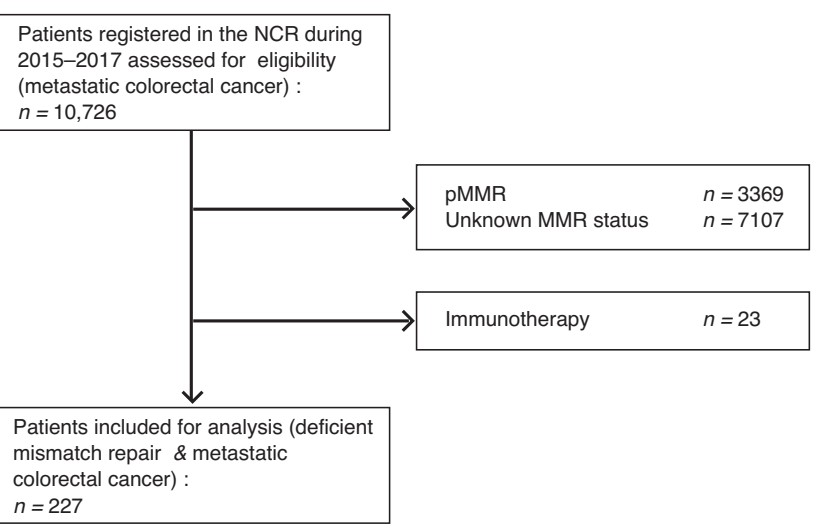

Fig. 1 Flow diagram of Netherlands Cancer Registry (NCR) patients. All NCR patients with histologically proven metastatic colorectal cancer (mCRC) were assessed for eligibility. Patients were excluded if mismatch repair (MMR) status was unknown or proficient MMR (pMMR) and if patients received immunotherapy during treatment. The final population-based cohort consists of 227 patients with deficient mismatch repair (dMMR) mCRC.

Netherlands are registered in the NCR. dMMR mCRC patients with an incidence date between January 1, 2015 and December 31, 2017 were included, since mismatch repair status is registered in the NCR for patients with an incidence date only after 2015. Figure 1 is a flow diagram of the patients identified in the NCR cohort. dMMR status was known when determined in routine clinical practice during the period of data registration. NCRderived patients include all synchronous and some metachronous mCRC patients with known dMMR status due to the data collection procedure of the NCR. For the same registration period, the overall survival and treatment status for PMMR mCRC NCRderived patients was collected.

\section{Data collection}

For population-based patients, pseudonymised clinical data on demographic characteristics, tumour characteristics and treatment information (type, response) were obtained from the NCR. Vital status for NCR patients was obtained using a yearly coupling with the municipal population registry to the cancer registry on February 1st, 2019. For NCR pMMR patients, clinical variables were obtained from the first registration period. For patients in the CAIRO trials, clinical data were available and follow-up information was updated up to October 2019. Data registration was completed for all dMMR patients, population-based and trial-based, by ensuring that no more than 30 days of clinical data was lacking prior to the vital status coupling. If data registration could not be completed, vital status was censored to the last known date of clinical data $(n=13)$.

Any $B R A F$ mutation detected was included in the definition of $B R A F$-mutant status. Sidedness of the primary tumour was defined as right-sided (coecum-transverse colon), left-sided (splenic flexure-sigmoid) and rectosigmoid/rectal. Antitumour therapy was defined as systemic treatment (excluding adjuvant therapy) or local treatment (surgical resection of metastases, radiofrequency ablation (RFA), microwave ablation (MWA), HIPEC (Hyperthermic Intraperitoneal Chemotherapy) or PIPAC (Pressurized Intra Peritoneal Aerosol Chemotherapy)). Antitumour therapy was categorised as follows: no antitumour therapy, local treatment only, local and systemic treatment and systemic treatment only.

Deficient mismatch repair

In the CAIRO trials dMMR status was determined according to the study protocol. ${ }^{1,16,17}$ In the NCR cohort, dMMR was determined according to Dutch guidelines in accredited laboratories, using immunohistochemistry and/or polymerase chain reaction.
Study parameters

Overall survival was defined as the interval from diagnosis of metastatic disease until death of any cause or date of last followup if alive (OS). In patients receiving systemic treatment, OS was measured from each treatment line initiation, resulting in OS1 in patients receiving first-line systemic treatment, and similarly OS2 and OS3 in patients receiving second-line or third-line systemic treatment starting from second-line or third-line initiation, respectively.

Survival rates and patient characteristics were obtained from published $\mathrm{ICI}$ trials. ${ }^{7,8}$ In order to bring our results in perspective with $\mathrm{ICl}$ trials without a control arm, our results were reported alongside the most comparable $\mathrm{ICl}$ trials, which analysed survival from second-line.

Statistical analysis

Baseline characteristics of the patients were analysed for the whole cohort and relevant differences between population-based and trial-based groups were described. Kaplan-Meier curves and 9-month and 12-month survival rate estimates were obtained for OS, OS1, OS2 and OS3. Subgroup analyses between populationbased and trial-based patients were performed using the logrank test.

Cox regression univariate and multivariable analysis was performed in patients receiving first-line treatment for OS. Ten preselected prognostic factors were selected: age at diagnosis of metastatic disease, gender, trial participation, BRAF mutation, primary tumour sidedness, metastatic sites location, stage at diagnosis, number of treatment lines given, primary tumour resection and metastasectomy. ${ }^{3,10,18}$ An unadjusted median overall survival (from diagnosis metastatic disease) for each level of the covariates was obtained by performing a log-rank test in patients receiving first-line systemic therapy. Multiple imputation by chained equations was used for covariates with missing data. ${ }^{19}$ From the complete dataset of variables, predictor variables with a correlation $>0.20$ with the missing variables and $<15 \%$ missing values were selected to use alongside the ten covariates and Cox regression outcome variables in multiple imputation. Patients with missing data were compared to patients with complete-cases (Supplementary Table S1). Univariate hazard ratios for each covariate were obtained using Cox regression. A stratified Cox proportional hazards multivariable model was obtained using the preselected ten covariates for OS; stratified for the number of treatment lines received since this covariate violated the proportional hazards assumption. Regression analysis was performed on each imputed dataset and combined using Rubin's rules.

All analyses were performed in R (version 3.5.1, "survival", "survminer", "mice" and "lattice" packages ${ }^{20}$ ).

\section{RESULTS}

Patient characteristics

The cohort comprises 281 patients: 227 population-based (NCR) and 54 trial-based patients. The characteristics of the cohort are described in Table 1.

Of the 281 patients, $57 \%$ were female, $73 \%$ had a right-sided tumour. Age had a bimodal distribution around 50 and 70 years. Of patients with a known BRAF mutation status, $55 \%(n=82 / 150)$ had a $B R A F$ mutation. A primary tumour resection and metastasectomy was performed in $78 \%$ and $23 \%$ of patients, respectively. Of patients with a known WHO performance score at start of firstline treatment, 93\% $(n=102 / 109)$ had a WHO performance score of $0-1$, with an unknown performance score in 64 patients.

In our cohort, $26 \%(n=72)$ of patients received no antitumour treatment, 13\% $(n=36)$ received local treatment, 14\% $(n=38)$ received local and systemic treatment and $48 \%(n=135)$ received only systemic treatment (Table 1). Sixty-two percent of patients 
Table 1. Patient characteristics.

\begin{tabular}{|c|c|}
\hline & $\begin{array}{l}\text { dMMR cohort } \\
n=281\end{array}$ \\
\hline \multicolumn{2}{|l|}{ Patient type } \\
\hline Trial-based & $54(19.2)$ \\
\hline Population-based & $227(80.8)$ \\
\hline \multicolumn{2}{|l|}{ Age (\%) } \\
\hline$\leq 55$ years & $59(21.1)$ \\
\hline $56-65$ years & $57(20.4)$ \\
\hline $66-75$ years & $103(36.9)$ \\
\hline$>75$ years & $60(21.5)$ \\
\hline Female (\%) & $159(56.6)$ \\
\hline \multicolumn{2}{|l|}{ BRAF mutational status (\%) } \\
\hline Wildtype & $68(45.3)$ \\
\hline Mutation & $82(54.7)$ \\
\hline Unknown & 131 \\
\hline \multicolumn{2}{|l|}{ RAS mutational status (\%) } \\
\hline Wildtype & $104(81.2)$ \\
\hline Mutation & $24(18.8)$ \\
\hline Unknown & 153 \\
\hline \multicolumn{2}{|l|}{ Stage $(\%)$} \\
\hline 1 & $2(0.7)$ \\
\hline II & $28(10.0)$ \\
\hline III & $88(31.5)$ \\
\hline IV & $161(57.7)$ \\
\hline \multicolumn{2}{|l|}{ Sidedness (\%) } \\
\hline Right-sided & $202(72.9)$ \\
\hline Left-sided & $55(19.9)$ \\
\hline Rectosigmoid/rectum & $20(7.2)$ \\
\hline Synchronous metastatic pattern (\%) & $194(69.0)$ \\
\hline \multicolumn{2}{|l|}{ Metastatic localisation (\%) } \\
\hline Liver-only & $61(21.7)$ \\
\hline Extrahepatic & $133(47.3)$ \\
\hline Peritoneal & $87(31.0)$ \\
\hline \multicolumn{2}{|l|}{ Number of metastatic sites (\%) } \\
\hline 1 & $166(59.3)$ \\
\hline 2 & $71(25.4)$ \\
\hline 3 & $33(11.8)$ \\
\hline$\geq 4$ & $10(3.6)$ \\
\hline Primary tumour resection (\%) & 219 (77.9) \\
\hline Metastasectomy (\%) & $64(22.8)$ \\
\hline \multicolumn{2}{|l|}{ Local treatment metastases (\%) } \\
\hline RFA & $6(2.3)$ \\
\hline MWA & $1(0.4)$ \\
\hline HIPEC & $27(9.6)$ \\
\hline \multicolumn{2}{|l|}{ Antitumour therapy (\%) } \\
\hline No treatment & $72(25.6)$ \\
\hline Local treatment & $36(12.8)$ \\
\hline Local and systemic treatment & $38(13.5)$ \\
\hline Systemic treatment & $135(48.0)$ \\
\hline Adjuvant chemotherapy (\%) & $47(16.7)$ \\
\hline \multicolumn{2}{|l|}{ Systemic therapy } \\
\hline First-line treatment (\%) & $173(61.6)$ \\
\hline Second-line treatment (\%) & $72(25.6)$ \\
\hline Third-line treatment (\%) & $21(7.5)$ \\
\hline
\end{tabular}

\begin{tabular}{|cc|}
\hline Table 1. continued & $\begin{array}{l}\text { dMMR cohort } \\
n=281\end{array}$ \\
\hline Fourth-line treatment (\%) & $3(1.1)$ \\
WHO PS (at start first-line, \% of first-line) & $57(52.2)$ \\
Score 0 & $45(41.3)$ \\
Score 1 & $7(6.4)$ \\
Score $\geq 2$ & 64 \\
Unknown & \\
\hline
\end{tabular}

Characteristics of patients at diagnosis of metastatic disease with treatment information during the course of disease. Trial-based patients were obtained from the CAIRO $(n=19)$, CAIRO2 $(n=31)$ and CAIRO3 $(n=$ 4) phase III randomised controlled trials. Sidedness of the primary tumour was defined as right-sided (coecum-transverse colon), left-sided (splenic flexure-sigmoid) and rectosigmoid/rectal. Local treatment was defined as metastasectomy or local metastatic treatment (RFA, MWA or HIPEC/PIPAC) and systemic treatment as all systemic therapy given for metastatic disease (excluding adjuvant therapy). Missing values are not shown if missing frequency was less than $5 \%$.

WHO PS: World Health Organisation Performance Score, percentages relative to amount of people receiving first-line treatment.

received first-line, $26 \%$ second-line and $8 \%$ third-line systemic non-immunotherapy. The type of treatment regimens and agents used per treatment line are described in Supplementary Table S2.

Comparing patient characteristics between population-based (2015-2018) and trial-based patients (2002-2011): populationbased patients were older at diagnosis of metastatic disease (age above 75 years in 24\% versus 10\%), had less primary tumour resections (74\% versus $96 \%)$ and more often resection of metastases (26\% versus 9\%), as shown in Supplementary Table S3. All trial-based patients were recruited from intervention trials with first-line systemic therapy, which is reflected in the proportion of patients receiving systemic therapy (100\% trial-based versus $52 \%$ population-based).

Follow-up

For the population-based and trial-based cohort, the median follow-up period from diagnosis of metastatic disease was 8.3 months (interquartile range [IQR] 3.1-17.6) and 16.8 months (9.5-22.8), respectively. At the end of the follow-up period, $70.1 \%$ of patients were deceased, which was an indication that follow-up was adequate. Follow-up was completed for 264 patients (94\%).

OS during treatment

The Kaplan-Meier survival curves from diagnosis of metastatic disease and from start of each therapy line are demonstrated in Fig. 2. We describe survival for patients who received antitumour therapy (systemic treatment, excluding adjuvant therapy, surgical resection of metastases and/or local treatment of metastases (including HIPEC or PIPAC)) versus patients who did not receive antitumour therapy. For patients who received antitumour therapy, median OS was 16.0 months (95\% Confidence Interval [C.I.] 13.8-19.6; $n=207$ ), whereas OS was 2.5 months (95\% C.I. $1.8-3.5 ; n=72$ ) in patients without antitumour therapy. Furthermore, examining survival per type of treatment received, median OS was longer in patients receiving local and systemic therapy (median OS 29.9 months, 95\% C.I. 17.9-not reached; $n=38$ ) compared to patients receiving only systemic therapy (13.9 months, 95\% C.I. 11.4-16.5; $n=133$ ), as shown in Supplementary Table S4. Compared to the other treatment categories, patients receiving local and systemic treatment more often had primary rectal tumours $(19 \%$ compared to $<7 \%$ in other categories), BRAF wildtype tumours (65\% compared to $<49 \%$ in 


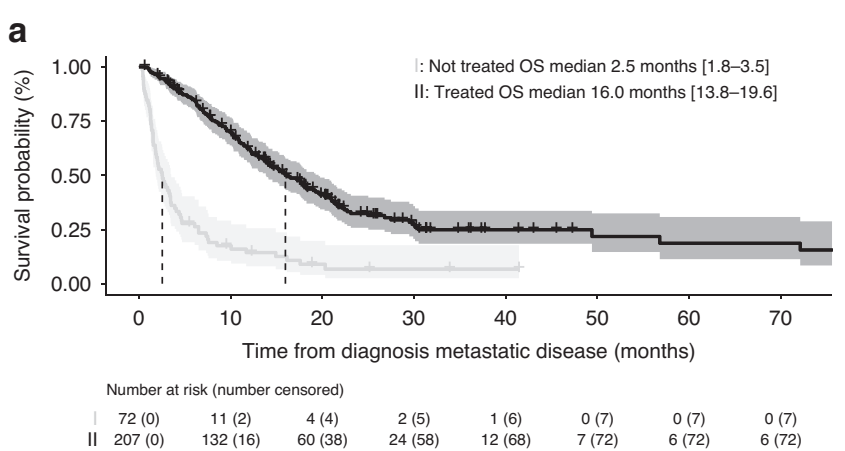

b
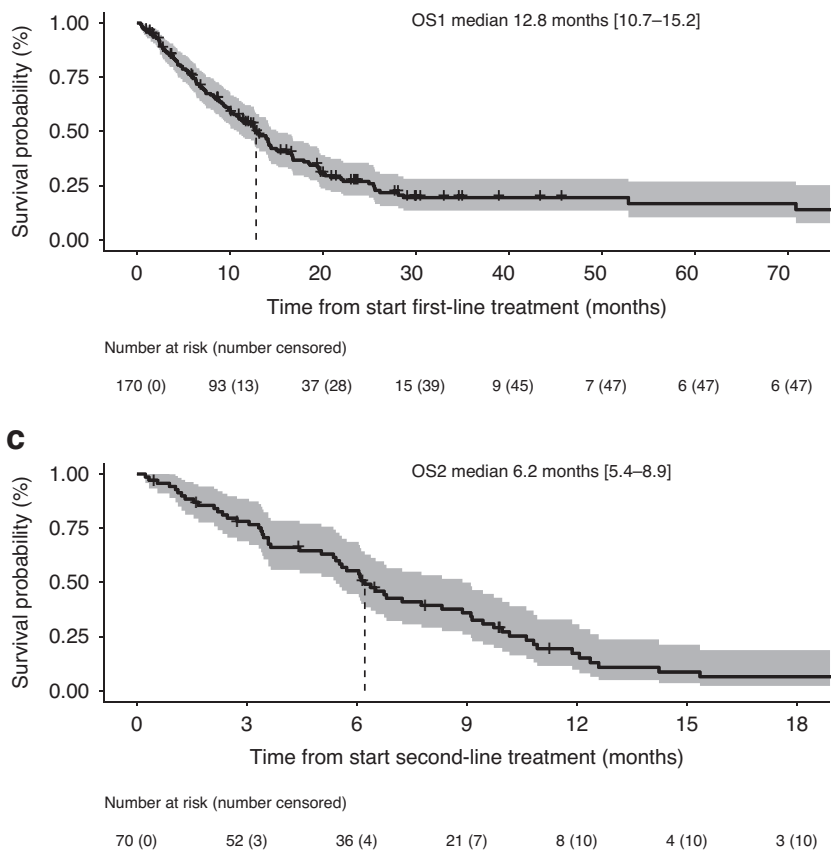

Fig. 2 OS, OS1 and OS2 in MCRC patients with dMMR. Predicted overall survival (OS) using Kaplan-Meier curves with confidence intervals, examined for the cohort as a whole and in patients who received systemic treatment. a OS from metastatic disease in the unselected dMMR cohort $(n=279)$, stratified by having received antitumour therapy $(n=207)$ versus no antitumour therapy $(n=72)$, b Overall survival from first-line systemic therapy initiation (OS1) in first-line patients $(n=170)$, c Overall survival from second-line systemic therapy initiation (OS2) in second-line patients $(n=70)$. The risk tables display the number of patients at risk and the number of censored patients. Median survival is indicated with a vertical dashed line in each plot and is indicated with $95 \%$ confidence interval lower and upper ranges in the legend.

other categories) and were younger ( $\leq 55$ years in $42 \%$ versus $<23 \%$ in other categories), as shown in Supplementary Table S5. Median OS increased in patients who received more systemic treatment lines (Supplementary Table S4).

In order to examine the OS from initiation of each treatment line, comprising only patients receiving the given treatment line, we examined the OS1, OS2 and OS3. The median overall survival from first-line systemic therapy initiation (OS1) was 12.8 months (95\% C.I. 10.7-15.2; $n=170$ ), from second-line systemic therapy initiation (OS2) 6.2 months (95\% C.I. 5.4-8.9; $n=70$ ) and from third-line systemic therapy initiation (OS3) 3.6 months (95\% C.I. 2.7-not reached; $n=19$ ).

From first-line systemic therapy initiation (OS1), estimated 9month and 12-month survival rates were $63.6 \%$ (95\% C.I. $56.6-71.5)$ and $53.8 \%$ (95\% C.I. 46.5-62.1). Similarly, from
Table 2. Overall survival in population-based metastatic colorectal cancer patients.

\begin{tabular}{llll}
\hline $\begin{array}{l}\text { Antitumour } \\
\text { therapy }\end{array}$ & $\begin{array}{l}\text { Mismatch } \\
\text { repair status }\end{array}$ & $\begin{array}{l}\text { Median OS } \\
\text { (months) }\end{array}$ & $p$ value \\
\hline Treated & dMMR $(n=155)$ & $16.0[13.0-22.1]$ & $<0.005$ \\
Untreated & pMMR $(n=2746)$ & $23.6[22.6-24.6]$ & \\
& dMMR $(n=72)$ & $2.5[1.8-3.5]$ & 0.005 \\
\hline & pMMR $(n=610)$ & $3.9[3.4-4.8]$ & \\
\hline
\end{tabular}

Population-based patients with metastatic colorectal cancer and known mismatch repair status, registered between 2015 and 2017 in the Netherlands Cancer Registry. OS was measured from diagnosis of metastatic disease until vital status coupling and is indicated with the 95\% confidence interval. Log-rank test was performed for untreated patients (dMMR versus pMMR) and for treated patients (dMMR versus pMMR). Antitumour therapy was defined as systemic treatment (with exception of adjuvant chemotherapy), metastasectomy or local metastatic treatment (RFA, MWA or HIPEC/PIPAC).

$d M M R$ deficient mismatch repair, HIPEC hyperthermic intraperitoneal chemotherapy, MWA microwave ablation, OS overall survival, PIPAC pressurised intraperitoneal aerosol chemotherapy, $P M M R$ proficient mismatch repair, RFA radiofrequency ablation.

second-line initiation (OS2), 9-month and 12-month survival rates were $35.9 \%$ (95\% C.I. 25.9-49.9) and 17.2\% (95\% C.I. 9.7-30.7). In patients who received second-line systemic treatment with a known WHO performance score $\leq 1$, similar to the inclusion criteria of the CheckMate 142 trials $(n=24),{ }^{7,8}$ predicted 9-month and 12month survival rates from start of second-line systemic therapy were $43.6 \%$ (95\% C.I. 27.4-69.4) and $17.4 \%$ (95\% C.I. 7.2-42.4), respectively. Supplementary Table $\mathrm{S} 6$ demonstrates the characteristics of our second-line patients alongside the nivolumab and nivolumab/ipilimumab CheckMate 142 trial cohorts.

We compared the survival between population-based and trialbased dMMR patients, examining differences in OS among treated patients, OS1 and OS2. There was no significant difference in logrank comparison of population-based versus trial-based patients in OS, OS1 or OS2. The median OS was 16.0 months (95\%C.I. $13.0-22.1 ; n=155)$ and 16.8 months (95\% C.I. 13.5-21.0; $n=52 ; p$ $=0.27$ ) in population-based and trial-based patients, respectively. Similarly, the median OS1 was 12.6 months (95\% C.I. 10.1-15.0; $n$ $=118$ population-based) and 13.5 months (95\% C.I. 9.1-19.6; $n=$ 52 trial-based; $p=0.59$ ). The median OS2 was 6.1 months (95\% C.I. 4.4-8.9) in 43 population-based patients and 6.7 months (95\% C.I. $5.0-10.2 ; p=0.58$ ) in 27 trial-based patients.

For population-based patients, we compared the median OS between patients with dMMR tumours versus pMMR tumours. For trial-based patients this was previously published, showing that the median OS was shorter in dMMR versus pMMR trial-based patients. ${ }^{1,17}$ For population-based patients, the median OS was significantly shorter in patients with $\mathrm{dMMR}$ tumours upon receiving antitumour therapy than pMMR tumours (Table 2). The median OS was 7.6 months shorter, with a median OS of 16.0 months (95\% C.I. 13.0-22.1; $n=155$ ) for dMMR tumours compared to 23.6 months (95\%C.I. 22.6-24.6; $n=2746 ; p<0.005$ ) for pMMR tumours in patients receiving treatment. In untreated patients, median OS was 2.5 months (95\% C.I. $1.8-3.5 ; n=72$ ) for dMMR tumours versus 3.9 months (95\% C.I. 3.4-4.8; $n=610 ; p=$ 0.005) for pMMR tumours.

Prognostic variables associated with overall survival in patients receiving first-line systemic treatment

In univariate analysis, metastasectomy and sidedness were significantly associated with OS from diagnosis of metastatic disease in patients receiving first-line systemic treatment (Table 3). $B R A F$ mutational status had a higher risk for shorter OS, albeit 
Table 3. Univariate and multivariable cox regression models for overall survival in first-line treated patients.

\begin{tabular}{|c|c|c|c|c|c|c|c|}
\hline Variable & Level & $n$ & \multicolumn{3}{|l|}{ Univariate } & \multicolumn{2}{|l|}{ Multivariable } \\
\hline \multirow[t]{2}{*}{ Age } & $\leq 65$ years & 83 & $18.0[13.9-22.4]$ & & & & \\
\hline & $>65$ years & 88 & $13.8[10.9-17.6]$ & $1.409(0.979-2.027)$ & 0.065 & $1.075(0.709-1.628)$ & 0.734 \\
\hline \multirow[t]{2}{*}{$B R A F$} & Wildtype & 63 & $19.6[14.6-22.7]$ & & & & \\
\hline & Mutation & 59 & 11.5 [8.7-17.9] & $1.509(0.998-2.282)$ & 0.052 & $1.610(0.936-2.771)$ & 0.086 \\
\hline \multirow[t]{3}{*}{ Metastatic sites } & Hepatic & 39 & $15.9[12.2-23.1]$ & & & & \\
\hline & Extrahepatic & 85 & $14.6[11.1-21.0]$ & $1.012(0.651-1.574)$ & 0.957 & $0.859(0.527-1.399)$ & 0.540 \\
\hline & Peritoneal & 47 & $15.2[12.3-21.6]$ & $1.017(0.601-1.721)$ & 0.950 & $0.814(0.447-1.484)$ & 0.502 \\
\hline Primary tumour resection & Yes & 126 & $16.5[13.5-21.0]$ & $0.664(0.437-1.007)$ & 0.054 & $0.628(0.365-1.083)$ & 0.094 \\
\hline \multirow[t]{2}{*}{ Primary tumour location } & Left-sided/rectosigmoid/rectum & 53 & $21.6[16.5-72.1]$ & & & & \\
\hline & Right-sided & 114 & $13.8[10.9-17.2]$ & $1.663(1.112-2.486)$ & $0.013^{a}$ & $1.705(1.042-2.790)$ & $0.034^{\mathrm{a}}$ \\
\hline \multirow[t]{3}{*}{ Stage at diagnosis } & Stage $1 / I I$ & 16 & 14.6 [10.3-NR] & & & & \\
\hline & Stage III & 39 & $14.6[7.1-20.8]$ & $1.293(0.655-2.550)$ & 0.459 & $0.944(0.447-1.996)$ & 0.881 \\
\hline & Stage IV & 113 & $16.0[13.1-19.8]$ & $0.980(0.532-1.805)$ & 0.948 & $0.723(0.351-1.490)$ & 0.380 \\
\hline \multirow[t]{2}{*}{ Cohort } & Population-based & 119 & $15.2[12.3-18.3]$ & & & & \\
\hline & Trial-based & 52 & $16.8[13.5-21.0]$ & $1.096(0.752-1.598)$ & 0.633 & $0.728(0.434-1.219)$ & 0.227 \\
\hline \multirow[t]{2}{*}{ Treatment lines received } & $<2$ & 100 & $13.8[9.9-19.6]$ & & & & \\
\hline & $\geq 2$ & 71 & $16.0[14.1-21.0]$ & $1.121(0.780-1.610)$ & 0.538 & & \\
\hline \multicolumn{8}{|c|}{$\begin{array}{l}\text { Multivariable results were calculated stratified per number of treatment lines received (which had violated the assumption of proportional hazards) using the } \\
\text { imputed dataset. An unadjusted median overall survival from diagnosis metastatic disease was obtained from a Kaplan-Meier curve stratified for the given } \\
\text { variable using the original dataset. The counts }(\mathrm{n}) \text { reflect the counts of the patients used in the survival analysis (non-imputed dataset), which may differ from } \\
\text { the total cohort due to missing data in the variable or outcome. } \\
\text { Bold numbers represent statistically significant hazard ratios. } \\
95 \% \text { C.I } 95 \% \text { confidence interval, CPH Cox Proportionate Hazards Model, HR hazard ratio, KM Kaplan-Meier, NR not reached, OS overall survival. } \\
\text { andicates statistically significant hazard ratio's ( } p \text { value }<0.05 \text { ). }\end{array}$} \\
\hline
\end{tabular}

nonsignificant, in univariate analysis (unadjusted hazard ratio [HR] 1.51 (95\% C.I. 1.00-2.28); $p=0.052$ ) as shown in Table 3.

The final multivariable model was a stratified Cox regression model for the number of treatment lines received ( $\leq 2$ and $>2$ ) since the variable violated the proportional hazards assumption. In the stratified Cox regression model for number of treatment lines received, metastasectomy is significantly associated with a longer survival (hazard ratio [HR] 0.49 (95\% C.I. 0.26-0.90); $p<0.05$ ) and right-sided tumour location is significantly associated with a shorter survival (HR1.71 (95\% C.I. 1.04-2.79); $p<0.05$ ) as shown in Table 3 . In patients receiving first-line systemic treatment, the unadjusted median OS was 29.5 months (95\% C.I. 17.9- not reached; $n=35$ ) and 14.1 months (95\% C.I. $11.5-17.2 ; n=136)$ for patients with and without a metastasectomy, respectively (Table 3). Similarly, the unadjusted median OS was 13.8 months (95\% C.I. $10.9-17.2 ; n=114)$ versus 21.6 months (95\% C.I. $16.5-72.1 ; n=53)$ in patients receiving first-line systemic treatment with a right-sided versus left-sided primary tumour location. BRAF mutational status had a higher risk for shorter OS, albeit nonsignificant, in multivariable analysis (HR1.61 (95\% C.I. $0.94-2.77) ; \quad p=0.086$ ), with an unadjusted median OS of 11.5 months (95\% C.I. 8.7-17.9; $n=59$ ) in patients receiving first-line systemic treatment with a BRAF-mutant tumour versus 19.6 months (95\% C.I. $14.6-22.7 ; n=63)$ in patients with a BRAF- wildtype tumour. The other covariates were not significantly associated with survival in the final multivariable model.

\section{DISCUSSION}

We present survival data of a large, comprehensive cohort of dMMR mCRC patients, not treated with immunotherapy. Our cohort offers a unique insight into the survival of dMMR mCRC patients while receiving systemic non-immunotherapy in first-, second- and third-line treatment. The OS in our dMMR mCRC cohort for all patients and patients receiving first-line treatment is comparable to previously reported survival data in dMMR mCRC patients without immunotherapy, including two population-based dMMR mCRC cohorts with a similar percentage of patients receiving systemic therapy. $5,9,10,12,21$ However, the OS in our dMMR mCRC patients is shorter than the median OS in three other publications, which ranged from 26-39 months.,11,22 The difference may be due to the patient characteristics in the cohorts, with cohorts including patients receiving immunother$a_{p y}{ }^{22}$ a high proportion (44-63\%) of Lynch syndrome (BRAF wildtype) patients, ${ }^{3,22}$ and a high proportion $(57 \%)$ of patients who underwent a metastasectomy. ${ }^{11}$ The median os from initiation of second-line treatment (6.2 months) in our cohort is drastically shorter than the recently reported median OS in 
second-line patients (21.6 months) in a cohort of dMMR mCRC patients receiving systemic non-immunotherapy and immunotherapy. ${ }^{22}$ The difference may be due to our cohort comprising only patients receiving systemic non-immunotherapy, while the Tougeron et al. dMMR mCRC patient cohort included patients receiving immunotherapy and a high proportion (44\%) of Lynch syndrome patients. ${ }^{22}$

In our population-based patients, the median OS during treatment was significantly shorter in dMMR compared to pMMR mCRC patients. This supports previous studies reporting a worse survival in $\mathrm{mCRC}$ patients with $\mathrm{dMMR}^{1,5,12}$ and is in contrast to studies showing a nonsignificant, null or opposite effect on survival. ${ }^{9,11,21,23}$ The conflicting results may lie in heterogeneity of the population cohort being studied, with studies finding a null or opposite effect on survival having included patients with a low percentage of BRAF mutations, ${ }^{9}$ only metachronous disease ${ }^{23}$ or younger patients. ${ }^{11}$ Our population reflects a clinically relevant cross-sectional population of Dutch patients who received MMR testing, indicating that patients with $\mathrm{mCRC}$ and known $\mathrm{dMMR}$ have a worse prognosis compared to pMMR patients.

In patients treated with at least one line of systemic treatment, we observed a significant association between metastasectomy with better survival and right-sided primary tumour location at diagnosis ('sidedness') with worse survival. In unselected mCRC patients and $\mathrm{dMMR}$ mCRC patients, metastasectomy is a known prognostic factor for OS.,10,24 Sidedness is an important prognostic factor in $\mathrm{mCRC}$ patients. However, sidedness has not yet been shown to be associated with OS in patients with dMMR tumours. ${ }^{3,24}$ Our results indicate that in dMMR mCRC patients, right-sidedness is associated with worse survival. Patients receiving first-line systemic treatment with a BRAF mutation had a higher risk for shorter survival in multivariable analysis, although nonsignificant, which is reflected with an 8-month difference in the unadjusted median OS in patients with a BRAF mutant versus $B R A F$-wildtype tumour. Studies have demonstrated that BRAF mutational status was prognostic within dMMR $\mathrm{mCRC}$ patients; ${ }^{10,23}$ however, this is not consistently shown. ${ }^{5,22}$ Although we did not identify a significant association for BRAF mutation with survival in patients receiving first-line systemic treatment, our results suggest that patients with a BRAF mutation do have a higher risk for shorter survival.

In addition to the prognostic factors which we examined in dMMR mCRC patients receiving first-line systemic treatment, other factors may contribute to the worse prognosis seen in dMMR mCRC patients. Population-based dMMR mCRC patients have a lower response rate to first-line systemic non-immunotherapy compared to pMMR mCRC patients ( $5 \%$ versus $44 \%$, respectively) and are also less likely to receive systemic therapy compared to pMMR mCRC patients (47\% versus $73 \%, p<0.001) .5,21$ Thus, the worse prognosis in dMMR $\mathrm{mCRC}$ patients is likely driven by several factors, potentially including primary tumour sidedness, BRAF mutational status, the response rate to systemic therapy, the ability to receive a metastatic resection, and other less well known factors, such as the PD-L1 gene expression level, reflecting immune evasion. ${ }^{25}$ Additionally, although dMMR mCRC patients are often analysed as one entity, different subgroups with different prognosis should be identified to compare survival results between studies including Lynch syndrome (often BRAFwildtype), sporadic $B R A F$ mutated dMMR tumours and sporadic $B R A F$-wildtype dMMR tumours. ${ }^{3,9,10}$ Although BRAF status and MMR status was known, due to unavailable data regarding MLH1 methylation and Lynch syndrome status we were unable to distinguish between sporadic versus Lynch origin.

We are aware of several limitations. Although we were able to include a broad range of relevant variables, we cannot exclude confounding from unmeasured variables. Secondly, our retrospective study design may have resulted in a selection of the population, since MMR status was not determined in all patients in daily practice. Lastly, comparison of our data with other studies may be confounded by differences in patient characteristics.

Our study is unique in providing survival data on a large cohort of population-based and trial-based dMMR mCRC patients in the pre-immunotherapy era. Our survival data of dMMR $\mathrm{mCRC}$ patients beyond first-line treatment may be compared with for instance the CheckMate 142 trial results, which examined nivolumab and nivolumab/ipilimumab treatment in dMMR mCRC patients beyond first-line treatment. ${ }^{7,8}$ The 9-month and 12month survival rates of patients receiving second-line treatment in our cohort of $35.9 \%$ and $17.2 \%$, respectively, are lower than the published 9-month survival rate in the nivolumab/ipilimumab arm of $87 \%$, the 12 -month survival rates in the nivolumab arm of $73 \%$ and the nivolumab/ipilimumab arm of $85 \% .^{7,8}$ The cohorts are comparable in key patient and tumour characteristics, although the immunotherapy cohorts were more heavily pretreated (40-54\% receiving $\geq 3$ treatment lines in the CheckMate 142 trials compared to $29 \%$ in our cohort) and patients more often having $B R A F$-wildtype status. Both characteristics may reflect a patient selection in the CheckMate 142 trials with a less aggressive clinical course compared to our cohort. Still, even the heavily treated patients in our cohort (who had received $\geq 3$ treatment lines) had a median OS of only 18 months. However, as we had no access to individual patient data of the other cohorts, a direct comparison between the cohorts was not possible. A comparison with the Phase 2 pembrolizumab trial was not possible due to the low number of patients in our cohort who received third-line treatment. $^{6}$ The CheckMate 142 results for patients receiving nivolumab/ipilimumab in first-line setting suggest a benefit of immunotherapy compared to our systemic non-immunotherapy first-line cohort, with a median 12 -month OS rate of $83 \%$ versus $54 \%$, respectively. ${ }^{26}$ This is supported by the Keynote- 177 Phase 3 randomised controlled trial results, which show that dMMR mCRC patients have a PFS benefit when receiving first-line pembrolizumab versus first-line systemic therapy (mFOLFOX6 or FOLFIRI combined with bevacizumab or cetuximab), with a median PFS of 16.5 months versus 8.2 months, respectively (HR0.60, 95\% C.I. $0.45-0.80, p=0.0002)^{27}$

In conclusion, we present survival data on a large, comprehensive cohort of dMMR mCRC patients, treated with or without systemic non-immunotherapy. Currently, available data from immunotherapy trials lack a control arm with standard systemic treatment. We demonstrate a poor prognostic value for dMMR in mCRC patients. Given the poor outcome in our dataset compared to the results of immunotherapy in dMMR mCRC patients, our data strongly support a survival benefit of immunotherapy in dMMR mCRC patients.

\section{ACKNOWLEDGEMENTS}

Part of the results were included in an abstract which was accepted for an oral presentation at the 2019 AACR Annual Meeting. ${ }^{28}$ The following oncologists and research nurses were involved in the study: M. Bax (Maxima Medical Center), M. Beusink (Amsterdam University Medical Center), C. Bresser-de Ruyter (Rode Kruis Hospital Beverwijk), S. Brouwer (Hospital Rijnstate), A. Cats (MD, Stichting Antoni van Leeuwenhoek Hospital), M. de Buck (Zorgsaam), H. de Graaf (MD, Medical Center Leeuwarden, Leeuwarden), M. Deelen (Diakonessenhuis Hospital Utrecht), $M$. Fellinger (Ziekenhuisgroep Twente), N. Golsteijn (Hospital Rivierenland), S.M. Hiddema (Groene Hart Hospital), D.F.S. Kehrer (MD, IJsselland Hospital), M. Laven (Catharina Hospital), H. Polderdijk (Haga Hospital), F. Ramakers (Zuyderland Medical Center), C.J. Rienks-Bosma (Medical Center Leeuwarden), R. Roukema (Antonius Hospital Sneek), S. Ruijgrok (IJsselland Hospital), J. Schellekens-van Bronswijk (Bravis Hospital), T. Simon (LangeLand Hospital), S. Sloof (Gelre Hospital), D.J. Storm (Spaarne Hospital), E. Valenteijn (Zaans Medical Center), S. van Broekhoven (Franciscus Gasthuis \& Vlietland), A.J. van de Vendel (Hospital Gelderse Vallei), E.J.E. van GestelWink (Meander Medical Center), M.-L. van Groesen (OLVG), I. van Rooij-Tieleman (VieCuri Medical Center), M. Vercoulen (Elkerliek Hospital), M.J. Weterman (Amsterdam University Medical Center, University of Amsterdam). We thank the registration team of the Netherlands Comprehensive Cancer Organisation (IKNL) for the 
collection of data for the Netherlands Cancer Registry as well as IKNL staff for scientific advice.

\section{AUTHOR CONTRIBUTIONS}

Study concepts and design were completed by G.E.W., M.A.G.E., A.M.M., P.A.H.H., C.J. A.P., G.R.V., J.M.L.R. and M.K. Data acquisition: all authors with the exception of A.M.M. and P.A.H.H. Quality control of data and algorithms, data analysis and interpretation, statistical analysis, manuscript preparation and editing were completed by G.E.W., M. A.G.E., A.M.M., C.J.A.P., G.R.V., J.M.L.R. and M.K. The manuscript was reviewed and approved by all authors.

\section{ADDITIONAL INFORMATION}

Ethics approval and consent to participate For trial-based patients, patient inclusion criteria, informed consent and study protocols for the trials were published previously. ${ }^{13-15}$ For population-based patients, pseudonymised clinical data on demographic characteristics, tumour characteristics and treatment information (type, response) were obtained from the Netherlands Cancer Registry. The privacy rights for patients were maintained. The study was performed in accordance with the Declaration of Helsinki.

Consent to publish not applicable.

Data availability The datasets generated during and analyzed during the current study are not publicly available due to the regulations of the Netherlands Cancer Registry but are available from the corresponding author or Netherlands Cancer Registry on reasonable request.

Competing interests The authors declare no conflict of interest. J.W.B.G. Institutional financial instructs (IFI): BMS, Pierre Fabre, Roche, MSD, Shire, Amgen; M.K. IFI: Amgen, Bayer, BMS, Merck-Serono, Nordic Farma, Roche, Servier, Sirtex, Sanofi-Aventis; Nonfinancial interests (NFI): advisory role ZON-MW, daily board member DCCG, P.I. PLCRC; CJAP IFI: Amgen, Roche; J.M.L.R. IFI: Servier, Merck, Bayer; T.v.V. NFI: Pfizer, Ipsen, Astellas, Roche, Bayer; H.V. IFI: Immunovo, Glycostem, Roche; G.R.V. IFI: Servier, Bayer, Merck, BMS, Lilly. All grants were unrelated to the study and paid to the individual's institution.

\section{Funding information None.}

Supplementary information is available for this paper at https://doi.org/10.1038/ s41416-020-01076-0.

Note This work is published under the standard license to publish agreement. After 12 months the work will become freely available and the license terms will switch to a Creative Commons Attribution 4.0 International (CC BY 4.0).

Publisher's note Springer Nature remains neutral with regard to jurisdictional claims in published maps and institutional affiliations.

\section{REFERENCES}

1. Venderbosch, S., Nagtegaal, I. D., Maughan, T. S., Smith, C. G., Cheadle, J. P., Fisher, D. et al. Mismatch repair status and BRAF mutation status in metastatic colorectal cancer patients: a pooled analysis of the CAIRO, CAIRO2, COIN, and FOCUS studies. Clin. Cancer Res. 20, 5322-5330 (2014).

2. Gelsomino, F., Barbolini, M., Spallanzani, A., Pugliese, G. \& Cascinu, S. The evolving role of microsatellite instability in colorectal cancer: a review. Cancer Treat. Rev. 51, 19-26 (2016)

3. Cohen, R., Buhard, O., Cervera, P., Hain, E., Dumont, S. \& Andre, T. Clinical and molecular characterisation of hereditary and sporadic metastatic colorectal cancers harbouring microsatellite instability/DNA mismatch repair deficiency. Eur. J. Cancer 86, 266-274 (2017).

4. Kim, C. G., Ahn, J. B., Jung, M., Beom, S. H., Kim, C., Kim, J. H. et al. Effects of microsatellite instability on recurrence patterns and outcomes in colorectal cancers. Br. J. Cancer 115, 25-33 (2016).

5. Aasebø, K., Dragomir, A., Sundström, M., Mezheyeuski, A., Edqvist, P. H., Eide, G. E. et al. Consequences of a high incidence of microsatellite instability and BRAFmutated tumors: a population-based cohort of metastatic colorectal cancer patients. Cancer Med. 8, 3623-3635 (2019).

6. Le, D. T., Uram, J. N., Wang, H., Bartlett, B. R., Kemberling, H., Eyring, A. D. et al. PD1 blockade in tumors with mismatch-repair deficiency. N. Engl. J. Med. 372, 2509-2520 (2015).
7. Overman, M. J., Mcdermott, R., Leach, J. L., Lonardi, S., Lenz, H., Morse, M. A. et al. Nivolumab in patients with metastatic DNA mismatch repair-deficient or microsatellite instability-high colorectal cancer (CheckMate 142): an open-label, multicentre, phase 2 study. Lancet 18, 1182-1191 (2017).

8. Overman, M. J., Lonardi, S., Yeung, K., Wong, M., Lenz, H., Gelsomino, F. et al. Durable clinical benefit with nivolumab plus ipilimumab in DNA mismatch repair-deficient/microsatellite instability-high metastatic colorectal cancer. J. Clin. Oncol. 36, 773-779 (2018).

9. Alex, A. K., Siqueira, S., Coudry, R., Santos, J., Alves, M., Hoff, P. M. et al. Response to chemotherapy and prognosis in metastatic colorectal cancer with DNA deficient mismatch repair. Clin. Colorectal Cancer 16, 228-239 (2017).

10. Goldstein, J., Tran, B., Ensor, J., Gibbs, P., Wong, H. L., Wong, S. F. et al. Multicenter retrospective analysis of metastatic colorectal cancer (CRC) with high-level microsatellite instability (MSI-H). Ann. Oncol. 25, 1032-1038 (2014).

11. Jin, Z., Sanhueza, C. T., Johnson, B., Nagorney, D. M., Larson, D. W., Mara, K. C., Harmsen, W. C. et al. Outcome of mismatch repair-deficient metastatic colorectal cancer: The Mayo Clinic Experience. Oncologist 23, 1083-1091 (2018).

12. Tran, B., Kopetz, S., Tie, J., Gibbs, P. \& Jiang, Z. Impact of BRAF mutation and microsatellite instability on the pattern of metastatic spread and prognosis in metastatic colorectal. Cancer Cancer 117, 4623-4632 (2011).

13. Koopman, M., Antonini, N. F., Douma, J., Wals, J., Honkoop, A. H., Erdkamp, F. L. G. et al. Sequential versus combination chemotherapy with capecitabine, irinotecan, and oxaliplatin in advanced colorectal cancer (CAIRO): a phase III randomised controlled trial. Lancet 370, 135-142 (2007).

14. Tol, J., Koopman, M., Rodenburg, C. J., Cats, A., Creemers, G. J., Schrama, J. G. et al. A randomised phase III study on capecitabine, oxaliplatin and bevacizumab with or without cetuximab in first-line advanced colorectal cancer, the CAIRO2 study of the Dutch Colorectal Cancer Group (DCCG). An interim analysis of toxicity. Ann. Oncol. 19, 734-738 (2008).

15. Simkens, L. H. J., Van Tinteren, H., May, A., Ten Tije, A. J., Creemers, G. J. M., Loosveld, O. J. L. et al. Maintenance treatment with capecitabine and bevacizumab in metastatic colorectal cancer (CAIRO3): a phase 3 randomised controlled trial of the Dutch Colorectal Cancer Group. Lancet 385, 1843-1852 (2015).

16. Koopman, M., Kortman, G. A. M., Mekenkamp, L., Ligtenberg, M. J. L., Hoogerbrugge, N., Antonini, N. F. et al. Deficient mismatch repair system in patients with sporadic advanced colorectal cancer. Br. J. Cancer 100, 266-273 (2009).

17. Goey, K. K. H., Elias, S. G., van Tinteren, H., Laclé, M. M., Willems, S. M., Offerhaus, G. J. A. et al. Maintenance treatment with capecitabine and bevacizumab versus observation in metastatic colorectal cancer: updated results and molecular subgroup analyses of the phase 3 CAIRO3 study. Ann. Oncol. 28, 2128-2134 (2017).

18. Sjoquist, K. M., Renfro, L. A., Simes, R. J., Tebbutt, N. C., Clarke, S., Seymour, M. T. et al. Personalizing survival predictions in advanced colorectal cancer: the ARCAD Nomogram Project. J. Natl Cancer Inst. 110, 638-648 (2018).

19. van Buuren, S., Groothuis-Oudshoorn, K., Vink, G., Doove, L., Jolani, S., Schouten, R. et al. mice: multivariate imputation by chained equations in R. R package. J. Stat. Softw. 45, 1-67 (2011).

20. R Core Team. R: a language and environment for statistical computing (R Foundation for Statistical Computing, Vienna, 2018).

21. Chong, L. C., Townsend, A. R., Young, J., Roy, A., Piantadosi, C., Hardingham, J. E. et al. Outcomes for metastatic colorectal cancer based on microsatellite instability: results from the South Australian metastatic colorectal cancer registry. Target Oncol. 14, 85-91 (2019).

22. Tougeron, D., Sueur, B., Zaanan, A., de la Fouchardiére, C., Sefrioui, D., Lecomte, T. et al. Prognosis and chemosensitivity of deficient MMR phenotype in patients with metastatic colorectal cancer: an AGEO retrospective multicenter study. Int. J. Cancer 147, 285-296 (2020).

23. Taieb, J., Shi, Q., Pederson, L., Alberts, S., Wolmark, N., Van Cutsem, E. et al. Prognosis of microsatellite instability and / or mismatch repair deficiency stage III colon cancer patients after disease recurrence following adjuvant treatment: results of an ACCENT pooled analysis of seven studies. Ann. Oncol. 30, 1466-1471 (2019).

24. Boeckx, N., Janssens, K., Van Camp, G., Rasschaert, M., Papadimitriou, K., Peeters, $M$. et al. The predictive value of primary tumor location in patients with metastatic colorectal cancer: a systematic review. Crit. Rev. Oncol. Hematol. 121, 1-10 (2018).

25. Marisa, L., Svrcek, M., Collura, A., Becht, E., Cervera, P., Wanherdrick, K. et al. The balance between cytotoxic T-cell lymphocytes and immune checkpoint expression in the prognosis of colon tumors. J. Natl Cancer Inst. 110, 68-77 (2018).

26. Lenz, H.-J., Lonardi, S., Zagonel, V., Van Cutsem, E., Limon, M. L., Yeung, K. et al Nivolumab plus low-dose ipilimumab as first-line therapy in microsatellite instability-high/DNA mismatch repair deficient metastatic colorectal cancer: Clinical update. J. Clin. Oncol. 38, abstr 11 (2020).

27. Andre, T., Shiu, K.-K., Kim, T. W., Jensen, B. V., Jensen, L. H., Punt, C. J. A. et al. Pembrolizumab versus chemotherapy for microsatellite instability-high/ 
mismatch repair deficient metastatic colorectal cancer: the phase 3 KEYNOTE-177 study. J. Clin. Oncol. 38, abstr LBA4 (2020).

28. Wensink, G. E., Elferink, M. A., May, A. M., Mol, L., Hamers, P. A., Punt, C. J. et al. Survival of patients with microsatellite instable (MSI) metastatic colorectal cancer
(mCRC) upon systemic non-immunotherapy [abstract]. Proceedings of the American Association for Cancer Research Annual Meeting 2019; 2019 Mar 29Apr 3; Atlanta, GA, Philad. Cancer Res. 79, 4467 (2019).

G. Emerens Wensink (iD), Marloes A. G. Elferink ${ }^{2}$, Anne M. May ${ }^{3}$, Linda Mol' ${ }^{2}$, Patricia A. H. Hamers ${ }^{1}$, Sandra D. Bakker ${ }^{4}$, Geert-Jan Creemers $^{5}$, Jan Willem B. de Groot ${ }^{6}$, Gerty J. de Klerk ${ }^{7}$, Brigitte C. M. Haberkorn ${ }^{8}$, Annebeth W. Haringhuizen ${ }^{9}$, Ronald Hoekstra ${ }^{10}$, J. Cornelis B. Hunting ${ }^{11}$, Emile D. Kerver ${ }^{12}$, Danielle Mathijssen-van Stein ${ }^{13}$, Marco B. Polée ${ }^{14}$, Johannes F. M. Pruijt ${ }^{15}$, Patricia Quarles van Ufford-Mannesse ${ }^{16}$, Sandra Radema ${ }^{17}$, Ronald C. Rietbroek ${ }^{18}$, Lieke H. J. Simkens ${ }^{19}$, Bea C. Tanis ${ }^{20}$, Daan ten Bokkel Huinink ${ }^{21}$, Manuel L. R. Tjin-A-Ton ${ }^{22}$, Cathrien S. Tromp-van Driel ${ }^{23}$, Monique M. Troost ${ }^{24}$, Agnes J. van de Wouw ${ }^{25}$, Franchette W. P. J. van den Berkmortel ${ }^{26}$, Anke J. M. van der Pas ${ }^{27}$, Ankie M. T. van der Velden ${ }^{28}$, Marjan A. van Dijk ${ }^{29}$, Joyce M. van Dodewaard-de Jong ${ }^{30}$, Edith B. van Druten ${ }^{31}$, Theo van Voorthuizen ${ }^{32}$, Gerrit Jan Veldhuis ${ }^{33}$, Henk M. W. Verheul ${ }^{34}$, Hanneke J. H. M. J. Vestjens ${ }^{25}$, Jeroen Vincent ${ }^{35}$, Onno W. Kranenburg $^{36,37}$, Cornelis J. A. Punt ${ }^{38}$, Geraldine R. Vink ${ }^{1,2}$, Jeanine M. L. Roodhart ${ }^{1}$ and Miriam Koopman (D)

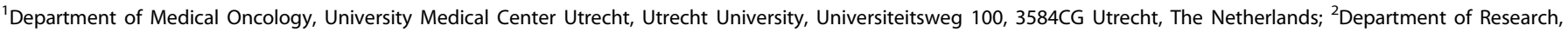

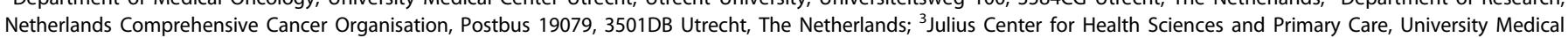
Center Utrecht, Utrecht University, P.O. Box 85500, 3508GA Utrecht, The Netherlands; ${ }^{4}$ Department of Medical Oncology, Zaans Medical Center, Postbus 210, 1500EE Zaandam,

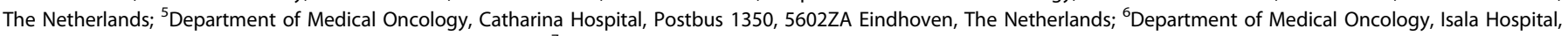

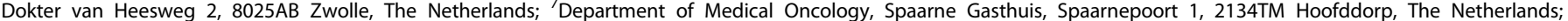

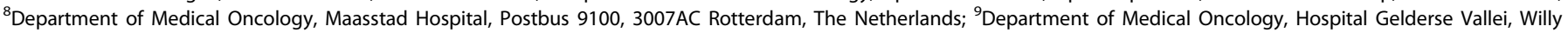

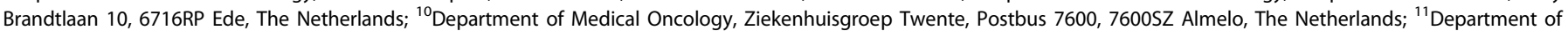

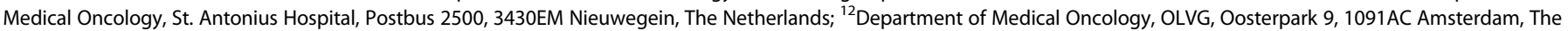

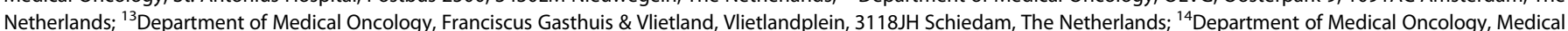

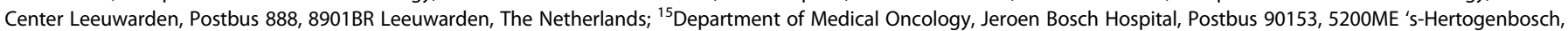

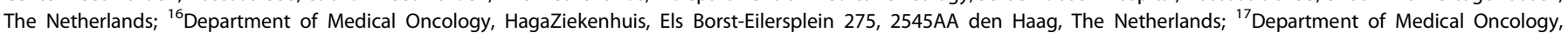

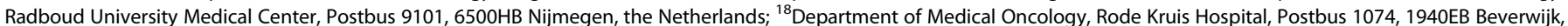

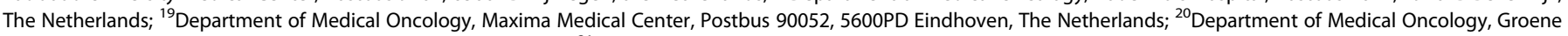

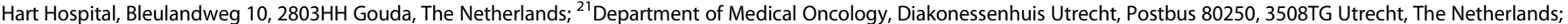

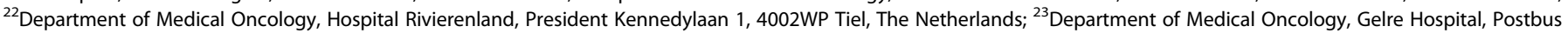

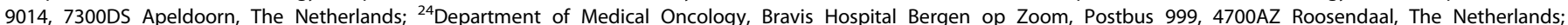

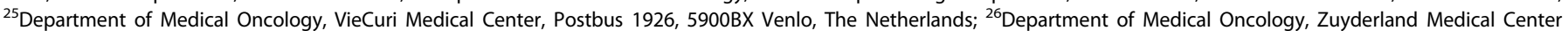

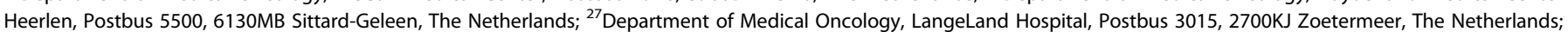

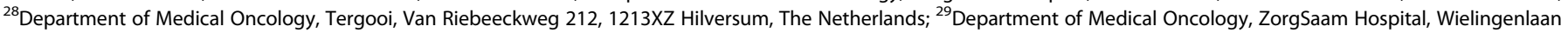

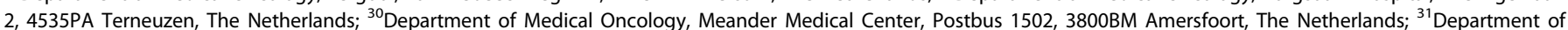

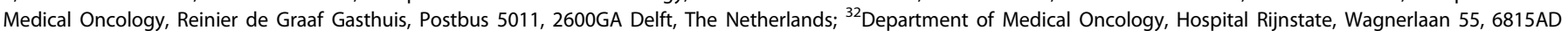

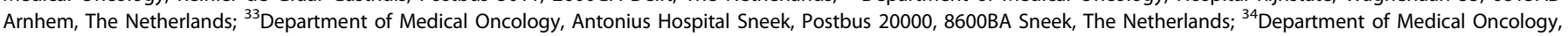

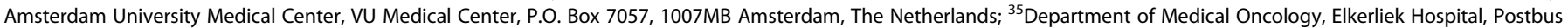

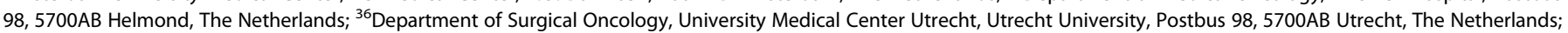

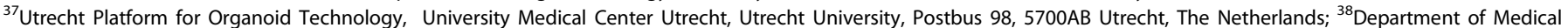
Oncology, Amsterdam University Medical Centers, University of Amsterdam, Postbus 22660, Amsterdam, The Netherlands. 